\title{
Equivalence of Particle-Particle Random Phase Approximation Correlation Energy and Ladder-Coupled-Cluster-Doubles
}

\author{
Degao Peng and Stephan N. Steinmann \\ Department of Chemistry, Duke University, \\ Durham, North Carolina, United States, 27708 \\ Helen van Aggelen \\ Department of Chemistry, Duke University, \\ Durham, North Carolina, United States, 27708
}

Ghent University, Department of Inorganic and Physical Chemistry, 9000 Ghent, Belgium

\author{
Weitao Yang \\ Department of Chemistry, Duke University, \\ Durham, North Carolina, United States, 27708 \\ Department of Physics, Duke University, \\ Durham, North Carolina, United States, 27708
}

\begin{abstract}
The recent proposal to determine the (exact) correlation energy based on pairing matrix fluctuations by van Aggelen et al. (arXiv:1306.4957) revived the interest in the simplest approximation along this path: the particle-particle random phase approximation (pp-RPA). In this paper, we present an analytical connection and numerical demonstrations of the equivalence of the correlation energy from particle-particle random phase approximation (pp-RPA) and ladder-coupledcluster-doubles (ladder-CCD). These two theories reduce to identical algebraic matrix equations and correlation energy expressions. The numerical examples illustrate that the correlation energy missed by pp-RPA in comparison with coupled-cluster singles and doubles is largely canceled out when considering reaction energies. This theoretical connection will be beneficial to design density functionals with strong ties to coupled-cluster theories and to study molecular properties at the pp-RPA level relying on well established coupled cluster techniques.
\end{abstract}




\section{INTRODUCTION}

The random-phase approximation (RPA) was originally proposed in the 1950s by Pine and Bohm[1, 2] to treat the homogeneous electron gas. Since then, the idea of RPA has spawned the studies of excitation energies, linear-response functions and correlation energies in solid state physics[3-6], nuclear physics[7-12], and quantum chemistry[13-16]. In the recent decade, there has been a renaissance of interest in the RPA correlation energy in quantum chemistry because of its good description of van der Waals interaction[16], the correct dissociation limit of $\mathrm{H}_{2}[17]$ and its perspective of the adiabatic connection in densityfunctional theory (DFT)[16]. These features have motivated the development of efficient implementations, leading to relatively low scaling algorithms $\left(O\left(N^{4} \log N\right)\right.$ by Eshuis et al.[18] and $O\left(N^{4}\right)$ by Ren et al.[19] with $N$ the number of basis functions). Correlation energy studies beyond RPA is an active field of research that achieves exciting results[20$26]$.

Recently, van Aggelen et al.[27] established an adiabatic connection for the exchangecorrelation energy in terms of the dynamic paring matrix fluctuation, parallel to the adiabatic connection fluctuation dissipation (ACFD) theorem in terms of the density fluctuation[5, 28]. Like the ACFD theorem, this adiabatic connection is in principle exact, but requires the particle-particle propagator as a function of the interaction strength. The particleparticle channel of random phase approximation (pp-RPA) is the first-order approximation to the paring matrix fluctuation. The first applications of the pp-RPA correlation energies to molecular systems provide promising results in describing systems with both fractional charge and fractional spin.[27] The RPA usually applied in quantum chemistry describes exclusively the particle-hole channel of correlations. To distinguish the two RPAs of different channels, we will, hereafter, refer to the conventional particle-hole RPA as ph-RPA. In nuclear physics, pp-RPA[7, 8, 29-37], also known as Brueckner's theory[38-41], is also widely discussed. In chemistry, however, the pp-RPA has only been used in computational study of Auger spectroscopy which involves double ionization of molecules[42, 43]. After finishing the development of Ref. [27] and this subsequent work, we became aware of an independent development by Scuseria et al.[44] that follows the same line of thought.

In the diagrammatic language extensively used in many-body perturbation-theory (MBPT), the ph-RPA correlation energy is the sum of all ring diagrams[7, 45]. Based on 
the same diagrammatic arguments, ph-RPA has been identified as a subset of the coupledcluster doubles (CCD) equations, i.e. accounting only for the ring summation terms[46]. Despite the well-known equivalence between the ph-RPA correlation energy and summation of all ring diagrams and the direct ring-CCD, the mathematical connection between the linear ph-RPA equation and the quadratic equation in direct ring-CCD has only recently been presented by Scuseria et al.[47], while ideas can be traced back to work done forty years before.[48] On the other hand, the pp-RPA correlation energy can be interpreted as the sum of all ladder diagrams[7]. As the sum of all ladder diagrams, the pp-RPA has also been referred to as the "ladder approximation" in the literature. Again, considering the diagrams involved, Čížek identified the sum of all ladder diagrams as a subset of CCD, which might be called ladder-CCD[46]. The pp-RPA wavefunction of an exponential form is textbook knowledge[8] with the argument of Thouless theorem[9] under the quasi-boson approximation. However, the authors are not aware of any explicit demonstration of the equivalence of the linear form of the pp-RPA equation and the quadratic ladder-CCD equation . The purpose of this paper is, following Ref. [47], to establish this connection between the two sets of seemingly distinct equations. The establishment of this connection might shed light on the relationship between Green's function based methods such as RPA and the coupled-cluster theory, an insight from which both fields could benefit. Furthermore, it is the authors' hope that the insight gained from linking Green's functions, coupled-cluster and density functional theory provides new stimulus to develop novel density functional approximations. Furthermore, the coupled-cluster connection opens up an direct way to obtain molecular properties from a virtual orbital dependent density functional.In the coupled cluster framework, the pp-RPA based excited states can straightforwardly be obtained via equation-of-motion coupled-cluster[49-52] or, equivalently, linear-response coupled-cluster theory[53, 54].

\section{THE PP-RPA EQUATION AND ITS STABILITY}

The pp-RPA equation can be derived from the two-particle Green's function, the equation-of-motion ansatz, or the linear-response time-dependent Hartree-Fock-Bogoliubov approximation (TDHFB)[7, 8, 27, 33]. The resulting generalized eigenvalue equation is very similar to the ph-RPA equation (see, for example, Ref. [7, 8, 16, 47] for the ph-RPA 
equation),

$$
\left[\begin{array}{cc}
\mathbf{A} & \mathbf{B} \\
\mathbf{B}^{\dagger} & \mathbf{C}
\end{array}\right]\left[\begin{array}{l}
\mathbf{x}_{n} \\
\mathbf{y}_{n}
\end{array}\right]=\omega_{n}\left[\begin{array}{cc}
\mathbf{I} & \mathbf{0} \\
\mathbf{0} & -\mathbf{I}
\end{array}\right]\left[\begin{array}{l}
\mathbf{x}_{n} \\
\mathbf{y}_{n}
\end{array}\right]
$$

where

$$
\begin{aligned}
& A_{a b, c d}=\left(\epsilon_{c}+\epsilon_{d}-2 \nu\right) \delta_{a c} \delta_{b d}+\langle a b \| c d\rangle, \\
& C_{i j, k l}=-\left(\epsilon_{k}+\epsilon_{l}-2 \nu\right) \delta_{k i} \delta_{j l}+\langle i j \| k l\rangle,
\end{aligned}
$$

and

$$
B_{a b, i j}=\langle a b|| i j\rangle
$$

We use indexes $i, j, k, l \ldots$ for occupied spin orbitals (holes), $a, b, c, d \ldots$ for unoccupied spin orbitals (particles), and $u, v, s, t \ldots$ for general spin orbitals. Furthermore, $m, n$ are used to denote eigenvector and eigenvalue indexes. Additionally, $\epsilon_{u}$ is the molecular orbital eigenvalue, and $\langle u v \| s t\rangle$ is the antisymmetrized two-electron integral

$$
\langle u v|| s t\rangle=\langle u v \mid s t\rangle-\langle u v \mid t s\rangle
$$

where

$$
\langle u v \mid s t\rangle=\sum_{\sigma_{1} \sigma_{2}} \int d \mathbf{r}_{1} d \mathbf{r}_{2} \frac{\phi_{u}^{*}\left(\mathbf{r}_{1} \sigma_{1}\right) \phi_{v}^{*}\left(\mathbf{r}_{2} \sigma_{2}\right) \phi_{s}\left(\mathbf{r}_{1} \sigma_{1}\right) \phi_{t}\left(\mathbf{r}_{2} \sigma_{2}\right)}{\left|\mathbf{r}_{1}-\mathbf{r}_{2}\right|} .
$$

(this is not true; when deriving the pp-RPA with a HF reference, you assume by definition a fixed electron number state, so the chemical potential is not stricly necessary; including it just guarantees some nice properties, like pos-definiteness. The role of the chemical potential is very similar in all derivations, EOM, TDHFB or GF.) . In practice, it is usually approximated to be half of HOMO (highest occupied molecular orbital) and LUMO (lowest unoccupied molecular orbital) eigenvalues[27]. We will later show that the exact choice of the chemical potential is unimportant within a certain range as long as the pp-RPA equation is stable.

The indexes of the matrix are either hole pairs or particle pairs. These indexes have only $i>j$ for hole pairs and $a>b$ for particle pairs to eliminate the redundancy. The number of particle (hole) pairs is

$$
N_{p p(h h)}=\frac{1}{2} N_{\operatorname{vir}(\text { occ })}\left(N_{\operatorname{vir}(\text { occ })}-1\right)
$$

where $N_{\text {vir(occ) }}$ is the number of virtual (occupied) orbitals. In general, $N_{p p}$ is much larger than $N_{h h}$. The dimension of the upper left (lower right) identity matrix in Eq. (1) is the 
same dimension of $\mathbf{A}(\mathbf{C})$. For the rest of the paper, the dimensions of identity matrices will be omitted as they are clear from the context. The difference of the dimensions of $\mathbf{A}$ and $\mathbf{C}$ makes the solution of the pp-RPA equation quite different from that of the usual phRPA equation or the linear-response time-dependent density-functional theory equation[55]. Nevertheless, Eq. 1 shares many conceptually similar properties to the ph-RPA equation as discussed in Ref. [10].

For simplicity, we use a compact matrix notation

$$
\mathbf{M} \mathbf{z}_{n}=\omega_{n} \mathbf{W} \mathbf{z}_{n}
$$

to denote Eq. (1), where $\mathbf{M}$ is the Hermitian matrix on the left hand side

$$
\mathbf{M}=\left[\begin{array}{cc}
\mathbf{A} & \mathbf{B} \\
\mathbf{B}^{\dagger} & \mathbf{C}
\end{array}\right]
$$

$\mathbf{W}$ is the non-positive definite metric

$$
\mathbf{W}=\left[\begin{array}{cc}
\mathbf{I} & \mathbf{0} \\
\mathbf{0} & -\mathbf{I}
\end{array}\right]
$$

and $\mathbf{z}_{n}$ is the full eigenvector

$$
\mathbf{z}_{n}=\left[\begin{array}{l}
\mathbf{x}_{n} \\
\mathbf{y}_{n}
\end{array}\right],
$$

with its eigenvalue $\omega_{n}$. Due to the non-positive definite metric $\mathbf{W}$, Eq. (1) is not guaranteed to have all real eigenvalues. We call $\mathbf{z}_{n}^{\dagger} \mathbf{W} \mathbf{z}_{n}$ the signature of an eigenvector $\mathbf{z}_{n}$. The signature can be positive, zero, or negative. The zero signature coincides with an imaginary eigenvalue (see Subsection A 1 in Appendix), while positive and negative signatures are associated with real eigenvalues. We categorize the eigenvectors according to their signature, where eigenvectors with positive signatures are called $N+2$ excitations and eigenvectors with negative signatures are called $N-2$ excitations. For a diagonalizable pp-RPA equation with all real eigenvalues, according to Subsection A 2 in Appendix, the orthonormalization of the eigenvectors can be written as,

$$
\mathbf{Z}^{\dagger} \mathbf{W Z}=\mathbf{W}
$$

with all $N+2$ eigenvectors to the left of all $N-2$ eigenvectors in $\mathbf{Z}$. This special arrangement will be kept all through the paper. 
When all the eigenvalues of a diagonalizable pp-RPA equation are real, the pp-RPA equation is defined to be stable if all the $N+2$ excitation eigenvalues are positive and $N-2$ excitation eigenvalues are negative, i.e. $\min _{n} \omega_{n}^{N+2}>0>\max _{m} \omega_{m}^{N-2}$. With the eigenvector arrangement according to signatures, the stability condition can be expressed in a concise equation,

$$
\operatorname{sign}(\boldsymbol{\omega})=\mathbf{W}
$$

where $\operatorname{sign}(\boldsymbol{\omega})$ is the sign function[56] of the eigenvalue matrix $\boldsymbol{\omega}$, which gives $[\operatorname{sign}(\boldsymbol{\omega})]_{n m}=$ $\delta_{n m} \operatorname{sign}\left(\omega_{n}\right)$ since $\boldsymbol{\omega}$ is diagonal. Note that Eq. (12) is a necessary but not sufficient condition for the stability of Eq. (13).

These eigenvalues are interpreted as the double ionization and double electron attachment energies in a molecular system, i.e.

$$
\omega_{n}^{N+2}=E_{n}^{N+2}-E_{0}^{N}-2 \nu,
$$

are the $N+2$ excitation energies, and

$$
\omega_{n}^{N-2}=E_{0}^{N}-E_{n}^{N-2}-2 \nu
$$

the $N-2$ excitation energies. With the eigenvalue interpretation of Eqs. (14)-(15), an unstable pp-RPA equation violates the energetic convexity condition[57]. It has not been proved that such stability is intrinsic for a self-consistent solution of a Hartree-Fock or Kohn-Sham/generalized Kohn-Sham molecular system, but in practice unstable solutions have never been encountered for molecular systems so far in Ref.[27] and in present work.

The stability condition of the pp-RPA equation is equivalent to the positive definiteness of the matrix M. See Subsection A 3 in Appendix for further details. The positive definiteness as the stability criterion has been used in Ref. [7].

With the whole spectrum of a stable pp-RPA equation, the pp-RPA correlation energy can be expressed in several equivalent ways[27] (you should refer to the literature here and to our paper, which has derivations in the appendix, because you give no derivations. I don't like to point out mistakes in other people's documents, and I would just refer to our paper without mentioning the mistake in Blaizot and Ripka, but that's my personal opinion).[77]

$$
E_{\mathrm{c}}^{\mathrm{pp}-\mathrm{RPA}}=\sum_{m} \omega_{m}^{N+2}-\operatorname{Tr} \mathbf{A}=-\sum_{n} \omega_{n}^{N-2}-\operatorname{Tr} \mathbf{C}=\frac{1}{2} \sum_{n}\left|\omega_{n}\right|-\frac{1}{2} \operatorname{Tr} \mathbf{M} .
$$


The precise value of $\nu$ is irrelevant for the correlation energy since it cancels out in the expression, Eq. 16, as long as

$$
\min _{m}\left(E_{m}^{N+2}-E_{0}^{N}\right)>2 \nu>\max _{n}\left(E_{0}^{N}-E_{n}^{N-2}\right),
$$

such that the $N+2$ eigenvalues are positive and the $N-2$ eigenvalues are negative. A proper chemical potential also categorizes $\mathbf{M}$ to be positive definite, an equivalent condition of the stability (see Subsection A 3 in Appendix for details).

\section{PROOF OF THE EQUIVALENCE OF PP-RPA AND LADDER-CCD}

The CCD ansatz, the simplest method in the coupled cluster family, expresses the wavefunction as

$$
|\mathrm{CCD}\rangle=e^{\hat{T}_{2}}\left|\Phi_{0}\right\rangle
$$

where $\left|\Phi_{0}\right\rangle$ is a single Slater determinant, and $\hat{T}_{2}$ is the two-body cluster operator

$$
\hat{T}_{2}=\frac{1}{2 !} \sum_{i j a b} t_{i j}^{a b} \hat{a}^{\dagger} \hat{i} \hat{b}^{\dagger} \hat{j}=\sum_{i j a b}^{i>j, a>b} t_{i j}^{a b} \hat{a}^{\dagger} \hat{i}^{\dagger} \hat{j},
$$

where $\hat{a}^{\dagger}, \hat{i}$ are the creation and annihilation operators for spin orbital $a$ and $i$, respectively and $t_{i j}^{a b}$ the double excitation amplitudes, having the symmetry

$$
t_{i j}^{a b}=-t_{j i}^{a b}=-t_{i j}^{b a}=t_{j i}^{b a}
$$

The correlation energy is expressed in terms of the amplitudes through the energy equation

$$
E_{\mathrm{c}}^{\mathrm{CCD}}=\sum_{i j a b}^{i>j, a>b}\langle i j \| a b\rangle t_{i j}^{a b},
$$

while the amplitudes $t_{i j}^{a b}$ are solved for by the CCD amplitude equation,

$$
\begin{aligned}
\left(\epsilon_{i}+\epsilon_{j}-\epsilon_{a}-\epsilon_{b}\right) t_{i j}^{a b}= & \langle a b \| i j\rangle+\frac{1}{2} \sum_{c d}\langle a b \| c d\rangle t_{i j}^{c d}+\frac{1}{2} \sum_{k l}\langle i j \| k l\rangle t_{k l}^{a b} \\
& -\sum_{k c}\left(\langle b k \| c j\rangle t_{i k}^{a c}-\langle b k \| c i\rangle t_{j k}^{a c}-\langle a k \| c j\rangle t_{i k}^{b c}+\langle a k \| c i\rangle t_{j k}^{b c}\right) \\
& +\sum_{k l c d}\langle k l \| c d\rangle\left[\frac{1}{4} t_{i j}^{c d} t_{k l}^{a b}-\frac{1}{2}\left(t_{i j}^{a c} t_{k l}^{b d}+t_{i j}^{b d} t_{k l}^{a c}\right)-\frac{1}{2}\left(t_{i k}^{a b} t_{j l}^{c d}+t_{i k}^{c d} t_{j l}^{a b}\right)+\left(t_{i k}^{a c} b_{j l}^{b d}+t_{i k}^{b d} t_{j l}^{a c}\right)\right] .
\end{aligned}
$$


Refer to Ref. [52] for details of the CCD equations.

By allowing only particle-hole summations in Eq. (21), Scuseria et al.[47] have shown that the amplitude equation reduces to the ph-RPA equation with exchange, i.e., the timedependent Hartree-Fock (TDHF) equation. Further eliminating the exchange term in the two-electron integral yields the conventional direct ph-RPA. Similarly, if we allow only summations of particle pairs and hole pairs, Eq. (21) becomes

$$
\begin{aligned}
& \sum_{k l}\left(\epsilon_{k}+\epsilon_{l}\right) t_{k l}^{a b} \delta_{k i} \delta_{j l}-\sum_{c d}\left(\epsilon_{c}+\epsilon_{d}\right) t_{i j}^{c d} \delta_{a c} \delta_{b d} \\
= & \langle a b \| i j\rangle+\frac{1}{2} \sum_{c d}\langle a b \| c d\rangle t_{i j}^{c d}+\frac{1}{2} \sum_{k l}\langle i j \| k l\rangle t_{k l}^{a b}+\frac{1}{4} \sum_{k l, c d} t_{k l}^{a b}\langle k l \| c d\rangle t_{i j}^{c d} .
\end{aligned}
$$

We refer to this restricted CCD as ladder-CCD, due to their inclusion of only ladder diagrams in the correlation energy. By utilizing the antisymmetry of the two-electron integrals $\langle u v \| s t\rangle=-\langle u v \| t s\rangle$, Eq. (22) can be rearranged as

$$
\sum_{c d}^{c>d} A_{a b, c d} t_{i j}^{c d}+\sum_{k l}^{k>l} C_{i j, k l} t_{k l}^{a b}+B_{a b, i j}+\sum_{k l, c d}^{k>l, c>d} t_{k l}^{a b} B_{c d, k l}^{*} t_{i j}^{c d}=0,
$$

with $A, B$, and $C$ defined in Eqs. (2)-(4). Denoting the amplitude as a matrix $T_{a b, i j}=t_{i j}^{a b}$, Eq. (23) results in an algebraic matrix equation

$$
\mathbf{A T}+\mathbf{T C}+\mathbf{B}+\mathbf{T B}^{\dagger} \mathbf{T}=0
$$

Now, we will show that the pp-RPA equation of Eq. (1) is equivalent to the ladder-CCD amplitude equation under the assumption that the pp-RPA equation is stable.

The pp-RPA equation for only the $N+2$ excitations reads,

$$
\left[\begin{array}{cc}
\mathbf{A} & \mathbf{B} \\
\mathbf{B}^{\dagger} & \mathbf{C}
\end{array}\right]\left[\begin{array}{l}
\mathbf{X} \\
\mathbf{Y}
\end{array}\right]=\left[\begin{array}{cc}
\mathbf{I} & \mathbf{0} \\
\mathbf{0} & -\mathbf{I}
\end{array}\right]\left[\begin{array}{c}
\mathbf{X} \\
\mathbf{Y}
\end{array}\right] \boldsymbol{\omega}^{N+2},
$$

where $\operatorname{dim} \mathbf{X}=N_{p} \times N_{p}, \operatorname{dim} \mathbf{Y}=N_{h} \times N_{p}$, and $\operatorname{dim} \boldsymbol{\omega}^{N+2}=N_{p} \times N_{p}$. Multiplying $\mathbf{X}^{-1}$ from the right on Eq. (25) gives

$$
\left[\begin{array}{cc}
\mathbf{A} & \mathbf{B} \\
\mathbf{B}^{\dagger} & \mathbf{C}
\end{array}\right]\left[\begin{array}{c}
\mathbf{I} \\
\tilde{\mathbf{T}}^{\dagger}
\end{array}\right]=\left[\begin{array}{cc}
\mathbf{I} & \mathbf{0} \\
\mathbf{0} & -\mathbf{I}
\end{array}\right]\left[\begin{array}{c}
\mathbf{I} \\
\tilde{\mathbf{T}}^{\dagger}
\end{array}\right] \mathbf{R}
$$

where

$$
\tilde{\mathbf{T}}=\left(\mathbf{Y X}^{-1}\right)^{\dagger}
$$


and

$$
\mathbf{R}=\mathbf{X} \boldsymbol{\omega}^{N+2} \mathbf{X}^{-1}
$$

The invertibility of $\mathbf{X}$ is guaranteed by a stable pp-RPA equation. See Subsection A 4 in Appendix for the detailed proof. Multiplying $\left[\tilde{\mathbf{T}}^{\dagger} \mathbf{1}\right]$ from the left to Eq. (26) results in

$$
\tilde{\mathbf{T}}^{\dagger} \mathbf{A}+\tilde{\mathbf{T}}^{\dagger} \mathbf{B} \tilde{\mathbf{T}}^{\dagger}+\mathbf{B}^{\dagger}+\mathbf{C} \tilde{\mathbf{T}}^{\dagger}=0
$$

Comparing Eq. (24) and Eq. (29), we infer that $\mathbf{T}=\tilde{\mathbf{T}}$.

The particle-particle block of Eq. (26) gives

$$
\mathbf{A}+\mathbf{B T}^{\dagger}=\mathbf{R}
$$

Then, the ladder-CCD correlation energy of Eq. (20) can be expressed as

$$
E_{\mathbf{c}}^{\text {ladder}-\mathbf{C C D}}=\operatorname{Tr}\left(\mathbf{B}^{\dagger} \mathbf{T}\right)=[\operatorname{Tr}(\mathbf{R}-\mathbf{A})]^{*}=\sum_{m} \omega_{m}^{N+2}-\operatorname{Tr} \mathbf{A}
$$

which is identical to the pp-RPA correlation energy in Eq. (16). From Eqs. (22)-(24), it is also clear that the chemical potential has no contribution because they cancel each other in the CCD equations through $\mathbf{A T}+\mathbf{T C}$.

Alternatively, one can also derive the equivalence using the $N-2$ excitation eigenvectors with similar techniques. The resulting amplitude will be the same, while the correlation energy expression will be the second equation in Eq. (16). An alternative proof of equivalence can also be formulated using a Schur decomposition in analogy to Appendix 5 in Ref. [47].

In conclusion, the correlation energy from pp-RPA is equivalent to that of ladder-CCD, assuming that the pp-RPA equation is stable. The exponential wavefunction of Eq. (17) with exponent of Eq. (27) has been proposed in Ref. [8], together with a similar form for ph-RPA, however without exploring their connection to the form of truncated CCD.

\section{NUMERICAL DEMONSTRATIONS}

All coupled cluster and second-order Møller-Plesset perturbation theory (MP2) computations reported herein are performed in a locally modified version of CFOUR[58], while pp-RPA is performed with QM4D[59].

Concerning the algorithm, truncating the CCD equations to include only the ladder diagrams (Eq. (22)) can be seen as a small modification of the CCD equations or a small 
extension of the linearized CCD, also known as CEPA(0) or D-MBPT $(\infty)$ [52], amplitude equations. Note that the computationally most expensive term of coupled-cluster singles and doubles (CCSD), scaling as $N_{\mathrm{occ}}^{2} N_{\mathrm{vir}}^{4}$, is the major part of the term quadratic in the amplitudes of Eq. (22). In terms of efficiency, the matrix multiplications necessary for solving the non-linear system of equations in standard coupled cluster algorithms are traded against the diagonalization in the pp-RPA algorithm, which, at the non-optimized stage of the code,[59] is significantly slower than solving the non-linear equations. However, the diagonalization has the indisputable advantage that the solution is unique, whereas the nonlinear coupled cluster equations have multiple minima (most of them lacking any physical meaning), without an a priori guarantee or check that the "correct" solution is found.[52]

All computations are carried out in the unrestricted Hartree-Fock (UHF) framework, but without breaking space symmetry. The correlation consistent basis sets of Dunning and coworkers[60,61] have been applied with cartesian d- and f- atomic-orbitals. The ladderCCD amplitudes are found to converge essentially as fast (or with a couple of iterations less) than the corresponding CCSD equations.

All total energies of ladder-CCD and pp-RPA (see Table I) agree exceedingly well, the largest difference being $10^{-5}$ Hartree, which is on the same order of magnitude as the difference in nuclear repulsion energy between the two programs and can have its origin in, e.g., integral screening (SCF and $\mathrm{CC}$ iteration convergence has been checked carefully). In terms of correlation energy, ladder-CCD captures between $43 \%$ (Be) to $80 \%$ (Ne) of CCSD, while the full CCD energy recovers about $99 \%$. Note that MP2 has min and max values of $70 \%$ and $99 \%$ for the same systems. Furthermore, changing to a DFT reference leads to an increased (in absolute terms) correlation energy, with $\min / \max$ values reaching $51(54) \%$ and $92(95) \%$ for B3LYP $[62,63]$ (PBE[64]) orbitals. It is important to point out that the present pp-RPA@DFT is not equivalent to ladder-CCD with a DFT reference when following the usual practice in the coupled cluster community[65, 66]. For pp-RPA@DFT, the molecular orbital energies are the eigenvalues of the Kohn-Sham Hamiltonian. However, the use of DFT orbitals in coupled cluster computations is considered as a "non-HF" reference wave function, for which the one-particle Hamiltonian is not diagonal and the corresponding terms are accounted for, yielding results that are much closer to HF based computations.[67, 68] 
Table I: Total energies of various methods. Geometries are taken from the G3 set[69, 70]. The basis set is cc-pVTZ, except for benzene where cc-pVDZ is applied. All energies are in Hartree.

\begin{tabular}{|c||c|c|c|c|c|c|c|c|}
\hline & HF & pp-RPA@HF & ladder-CCD & pp-RPA@PBE & pp-RPA@B3LYP & MP2 & CCD & CCSD \\
\hline \hline $\mathrm{He}$ & -2.861154 & -2.885608 & -2.885608 & -2.889343 & -2.888504 & -2.894441 & -2.900328 & -2.900351 \\
\hline $\mathrm{Li}$ & -7.432706 & -7.443903 & -7.443903 & -7.444664 & -7.444450 & -7.446781 & -7.449184 & -7.449243 \\
\hline $\mathrm{Be}$ & -14.572875 & -14.598923 & -14.598923 & -14.605231 & -14.603533 & -14.614751 & -14.632242 & -14.632817 \\
\hline $\mathrm{B}$ & -24.532104 & -24.566435 & -24.566436 & -24.575674 & -24.573063 & -24.584950 & -24.604746 & -24.605490 \\
\hline $\mathrm{C}$ & -37.691663 & -37.746778 & -37.746778 & -37.760145 & -37.756583 & -37.769564 & -37.789208 & -37.789809 \\
\hline $\mathrm{N}$ & -54.400883 & -54.482916 & -54.482916 & -54.500883 & -54.496235 & -54.509992 & -54.525553 & -54.525893 \\
\hline $\mathrm{O}$ & -74.811910 & -74.933839 & -74.933839 & -74.959853 & -74.953384 & -74.969918 & -74.985506 & -74.986128 \\
\hline $\mathrm{F}$ & -99.405657 & -99.576884 & -99.576884 & -99.611587 & -99.603292 & -99.622736 & -99.633484 & -99.634177 \\
\hline $\mathrm{Ne}$ & -128.532010 & -128.760771 & -128.760771 & -128.804849 & -128.794546 & -128.816523 & -128.817814 & -128.818536 \\
\hline $\mathrm{CH}_{4}$ & -40.213408 & -40.372051 & -40.372054 & -40.411910 & -40.402169 & -40.432266 & -40.452031 & -40.452991 \\
\hline $\mathrm{H}_{2} \mathrm{O}$ & -76.056687 & -76.266046 & -76.266049 & -76.318304 & -76.305731 & -76.336459 & -76.340863 & -76.342084 \\
\hline $\mathrm{NH}_{3}$ & -56.217964 & -56.404439 & -56.404440 & -56.452289 & -56.440556 & -56.471921 & -56.483441 & -56.484474 \\
\hline $\mathrm{CH}_{2} \mathrm{O}$ & -113.910280 & -114.227562 & -114.227552 & -114.313824 & -114.293495 & -114.341669 & -114.347547 & -114.351726 \\
\hline $\mathrm{C}_{6} \mathrm{H}_{6}$ & -230.722701 & -231.315273 & -231.315273 & -231.508132 & -231.460711 & -231.540504 & -231.571751 & -231.577366 \\
\hline
\end{tabular}

As a graphical illustration, Figure 1a shows the case of a dissociating cationic dimer $\left(\mathrm{Ne}_{2}^{+}\right)$, a typical probe for (de)localization error. We are using a spatial symmetry $\left(D_{\infty h}\right)$ preserving unrestricted $\mathrm{HF}$ reference wave function for $\mathrm{Ne}_{2}^{+}$. Again, the total energies of ladder-CCD and pp-RPA are identical to numerical precision (considering the two very different algorithms and programs), but not in very good agreement with CCSD. To further investigate the (de)localization error[71], Figure 1b shows the binding energy with respect to the separated fragments. The binding energy of ladder-CCD is in fairly good agreement with CCSD and only a small "bump" is observed somewhere between 3 and $4 \AA$, revealing that the missing absolute correlation energies in ladder-CCD compared to CCSD are almost irrelevant for the binding energy. The localization error of HF is over-corrected by MP2, but increasing the correlation treatment to the coupled cluster level improves the dissociation 
Table II: Atomization energies (in $\mathrm{kcal} \mathrm{mol}^{-1}$ ) of various methods. Geometries are taken from the G3 set[69, 70]. Experimental atomization energies are taken from Ref. [73-76].

The basis set is cc-pVTZ. The mean absolute deviation (MAD) is with respect to experimental data.

\begin{tabular}{|l||l|l|l|l|l|l|l|l|l|}
\hline & HF & pp-RPA@HF & ladder-CCD & pp-RPA@PBE & pp-RPA@B3LYP & MP2 & CCD & CCSD & Exp. \\
\hline \hline $\mathrm{CH}_{4}$ & 327.9 & 392.8 & 392.8 & 410.7 & 406.4 & 416.3 & 416.4 & 416.6 & 419.2 \\
\hline $\mathrm{H}_{2} \mathrm{O}$ & 153.8 & 208.7 & 208.7 & 225.8 & 221.7 & 230.3 & 223.2 & 223.6 & 232.2 \\
\hline $\mathrm{NH}_{3}$ & 199.3 & 264.9 & 264.9 & 284.5 & 279.8 & 290.2 & 287.7 & 288.1 & 297.5 \\
\hline $\mathrm{CH}_{2} \mathrm{O}$ & 255.5 & 343.5 & 343.5 & 373.5 & 366.8 & 378.1 & 359.7 & 361.6 & 373.6 \\
\hline \hline MAD & 96.5 & 28.2 & 28.2 & 7.0 & 12.0 & 4.2 & 8.9 & 8.2 & - \\
\hline
\end{tabular}

limit further, leading to the previously reported[27] negligible fractional charge error. Note that the ground state of $\mathrm{Ne}_{2}^{+}$is indeed ${ }^{2} \Sigma_{g}$, as compared to $\mathrm{F}_{2}^{+}$the ground state of which is ${ }^{2} \Pi_{g}[72]$. The influence of references with different symmetries (and therefore possibly spin-contamination) will be investigated in further studies.

Similarly to the binding energy of $\mathrm{Ne}_{2}^{+}$, the atomization energies (Table II) illustrate that the correlation energy missing in ladder-CCD largely cancels out when computing reaction energies. For the four molecules considered, ladder-CCD provides $77 \%$ on of the correction between the HF and CCSD atomization energies on average. This is to be compared with MP2 which recovers on average 107\%. However, the mean absolute deviation for ppRPA@PBE compared to the experimental values is substantially better, having the same level of accuracy of CCSD. In summary, the numerical analysis shows that ladder-CCD and pp-RPA are equivalent and that pp-RPA covers a substantial amount of correlation energy that is relevant for atomization energies of typical small molecules in Table II. An efficient pp-RPA implementation has, therefore, the potential to become a valuable electronic structure theory. 


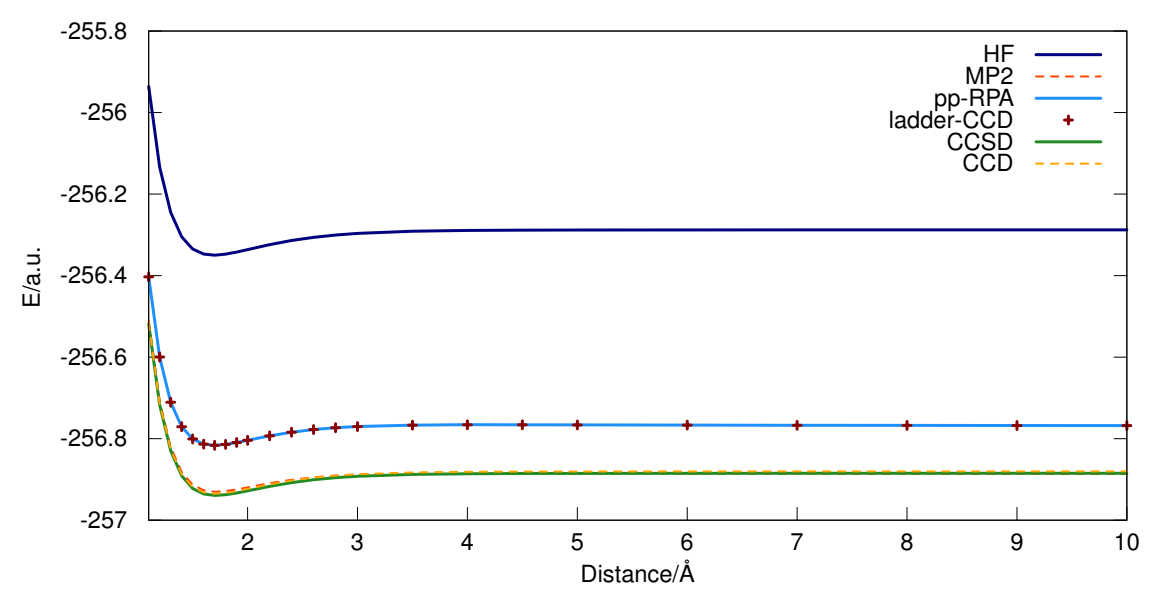

(a) The potential energy surface of $\mathrm{Ne}_{2}^{+}$

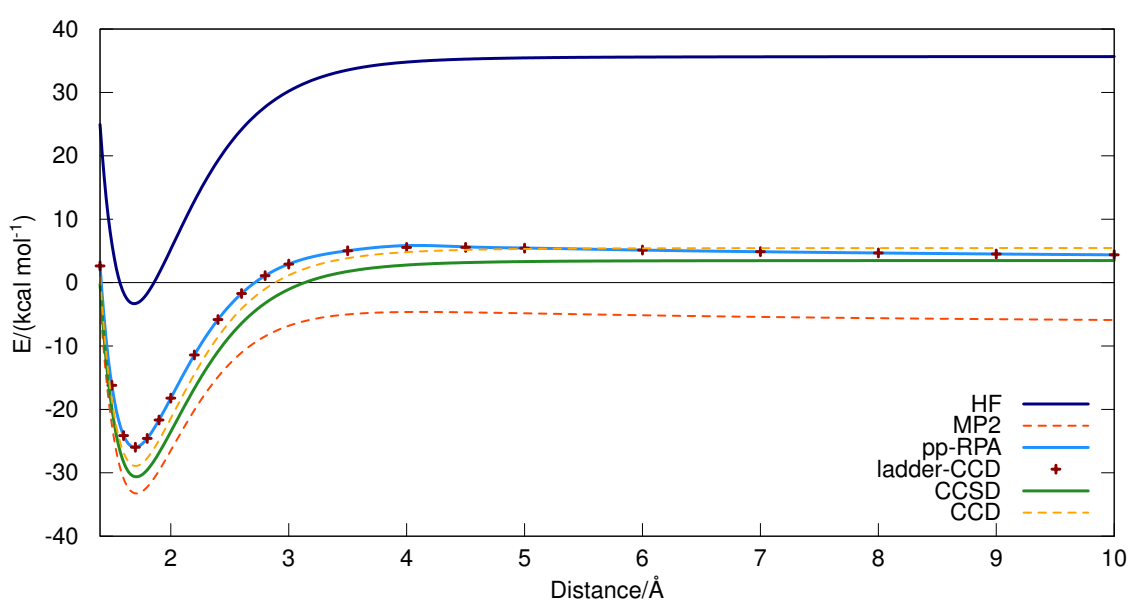

(b) The binding curve of $\mathrm{Ne}_{2}^{+}$(with respect to $\mathrm{Ne}$ and $\mathrm{Ne}^{+}$)

Figure 1: The potential energy surface (a) and the binding curve (b) of $\mathrm{Ne}_{2}^{+}$of various methods with basis set aug-cc-pVTZ. The total energies of pp-RPA are substantially overestimated (a), since the correlation energy of the ladder diagrams is not very well balanced (MP2 total energies are, on the scale of the figure, indistinguishable from CCD, and pp-RPA is correct through second order[27]). However, the binding energy (b) reveals that the missing correlation energy cancels almost perfectly out, yielding a pp-RPA binding energy curve very close to CCD, while MP2 deviates from CCSD in the other direction (overbinding). 


\section{CONCLUSIONS}

The connection between the linear pp-RPA equation and the quadratic ladder-CCD equation has been established and numerically verified. The numerical assessment suggests that pp-RPA is fairly accurate for some reaction energies, despite its incomplete diagram summation. This mathematical connection is helpful in understanding the relationship between Green's function based and the coupled-cluster methods. The ladder-CCD perspective of the pp-RPA makes the study of its ground and excited state properties straightforward.

\section{Acknowledgment}

Support from the Office of Naval Research (ONR) (N00014-09-1-0576), and the National Science Foundation (NSF) (CHE-09-11119) is gratefully appreciated. D.P. has also been supported by the William Krigbaum and Marcus Hobbs Fellowship from Duke University. S.N.S. acknowledges the Swiss NSF fellowship PBELP2_143559. H.v.A. appreciates the support form the FWO-Flanders (Scientific Research Fund Flanders). Discussions with Jianfeng Lu have been helpful.

[1] D. Bohm and D. Pines, Phys. Rev. 82, 625 (1951).

[2] D. Pines and D. Bohm, Phys. Rev. 85, 338 (1952).

[3] J. Lindhard, K. Dan. Vidensk. Selsk. Mat. Fys. Medd. 28, 8 (1954).

[4] A. G. Eguiluz, Phys. Rev. Lett. 51, 1907 (1983).

[5] D. C. Langreth and J. P. Perdew, Solid State Commun. 17, 1425 (1975).

[6] G. F. Giuliani and G. Vignale, Quantum Theory Of The Electron Liquid (Cambridge University Press, 2005).

[7] J. Blaizot and G. Ripka, Quantum theory of finite systems (Cambridge, MA, 1986).

[8] P. Ring and P. Schuck, The Nuclear Many-Body Problem (Springer, 2004), ISBN 9783540212065, URL http://books.google.com/books?id=PTynSM-nMA8C.

[9] D. Thouless, Nuclear Physics 21, 225 (1960), ISSN 00295582, URL http://linkinghub. elsevier.com/retrieve/pii/0029558260900481. 
[10] D. D. Thouless, Nuclear Physics 22, 78 (1961), ISSN 00295582, URL http://linkinghub. elsevier.com/retrieve/pii/0029558261903649http://www.sciencedirect.com/ science/article/pii/0029558261903649.

[11] D. Thouless and J. Valatin, Nuclear Physics 31, 211 (1962), ISSN 00295582, URL http: //linkinghub.elsevier.com/retrieve/pii/0029558262907411.

[12] E. R. Marshalek and J. Weneser, Ann. Phys. 53, 569 (1969).

[13] H. Eshuis, J. Yarkony, and F. Furche, J. Chem. Phys. 132, 234114 (2010).

[14] F. Furche, J. Chem. Phys. 129, 114105 (2008).

[15] S. Kurth and J. P. Perdew, Phys. Rev. B 59, 10461 (1999).

[16] F. Furche, Phys. Rev. B 64, 195120 (2001).

[17] M. Fuchs, Y.-M. Niquet, X. Gonze, and K. Burke, J. Chem. Phys. 122, 94116 (2005).

[18] H. Eshuis and F. Furche, J. Chem. Phys. 136, 84105 (2012).

[19] X. Ren, P. Rinke, C. Joas, and M. Scheffler, Journal of Materials Science 47, 7447 (2012), ISSN 0022-2461, URL http://link.springer.com/10.1007/s10853-012-6570-4.

[20] F. Furche and T. Van Voorhis, J. Chem. Phys. 122, 164106 (2005).

[21] W. Zhu, J. Toulouse, A. Savin, and J. G. Angyán, J. Chem. Phys. 132, 244108 (2010), ISSN 1089-7690, URL http://www.ncbi.nlm.nih.gov/pubmed/20590182.

[22] J. Toulouse, W. Zhu, J. G. Ángyán, and A. Savin, Phys. Rev. A 82, 032502 (2010), ISSN 1050-2947, URL http://link.aps.org/doi/10.1103/PhysRevA.82.032502.

[23] A. Hesselmann and A. Görling, Mol. Phys. 109, 2473 (2011).

[24] M. Hellgren, D. R. Rohr, and E. K. U. Gross, J. Chem. Phys. 136, 34106 (2012).

[25] P. Mori-Sánchez, A. J. Cohen, and W. Yang, Phys. Rev. A 85, 42507 (2012), ISSN 1050-2947, URL http://link.aps.org/doi/10.1103/PhysRevA.85.042507.

[26] T. Gould and J. F. Dobson, J. Chem. Phys. 138, 14109 (2013).

[27] H. van Aggelen, Y. Yang, and W. Yang (2013), 1306.4957, URL http://arxiv.org/abs/ 1306.4957.

[28] O. Gunnarsson and B. Lundqvist, Phys. Rev. B 13, 4274 (1976), URL http://prb.aps.org/ abstract/PRB/v13/i10/p4274\_1.

[29] N. Fukuda, F. Iwamoto, and K. Sawada, Phys. Rev. 135, A932 (1964).

[30] J. Toivanen and J. Suhonen, Phys. Rev. Lett. 75, 410 (1995).

[31] W. J. Mulhall, R. J. Liotta, J. A. Evans, and R. P. Perazzo, Nucl. Phys. A 93, 261 (1967). 
[32] D. J. Rowe, Phys. Rev. 175, 1283 (1968).

[33] D. J. Rowe, Rev. Mod. Phys. 40, 153 (1968).

[34] G. Ripka and R. Padjen, Nucl. Phys. A 132, 489 (1969).

[35] J. Vary and J. N. Ginocchio, Nucl. Phys. A 166, 479 (1971).

[36] G. Blanchon, N. V. Mau, A. Bonaccorso, M. Dupuis, and N. Pillet, Phys. Rev. C 82, 34313 (2010).

[37] J. C. Pacheco and N. Vinh Mau, Phys. Rev. C 65, 44004 (2002).

[38] K. A. Brueckner and C. A. Levinson, Phys. Rev. 97, 1344 (1955), ISSN 0031899X, URL http://link.aps.org/doi/10.1103/PhysRev.97.1344http://prola.aps.org/abstract/ PR/v97/i5/p1344\_1.

[39] R. Eden, Proceedings of the Royal Society A 235, 408 (1956), URL http://rspa. royalsocietypublishing.org/content/235/1202/408. short.

[40] H. Bethe, Phys. Rev. 103, 1353 (1956), ISSN 0031-899X, URL http://prola.aps.org/ abstract/PR/v103/i5/p1353\_1http://link.aps.org/doi/10.1103/PhysRev.103.1353.

[41] J. Goldstone, Proceedings of the Royal Society of ... 239, 267 (1957), URL http://rspa. royalsocietypublishing.org/content/239/1217/267. short.

[42] C.-M. Liegener, J. Chem. Phys. 104, 2940 (1996), ISSN 00219606, URL http://link.aip. org/link/JCPSA6/v104/i8/p2940/s1\\&Agg=doi.

[43] C.-M. Liegener, Chem. Phys. Lett. 90, 188 (1982), ISSN 00092614, URL http://linkinghub. elsevier.com/retrieve/pii/0009261482800225.

[44] G. E. Scuseria, T. M. Henderson, and I. W. Bulik, pp. 1-27 (2013), 1306.6360, URL http: //arxiv.org/abs/1306.6360.

[45] M. Gell-Mann and K. Brueckner, Phys. Rev. 106, 364 (1957), ISSN 0031-899X, URL http: //link.aps.org/doi/10.1103/PhysRev.106.364.

[46] J. Čížek, J. Chem. Phys. 45, 4256 (1966), ISSN 00219606, URL http://link.aip.org/link/ ?JCP/45/4256/1 \\&Agg=doi.

[47] G. E. Scuseria, T. M. Henderson, and D. C. Sorensen, J. Chem. Phys. 129, 231101 (2008), ISSN 1089-7690, URL http://www.ncbi.nlm.nih.gov/pubmed/19102519.

[48] E. Sanderson, Physics Letters 19, 141 (1965), ISSN 00319163, URL http://dx.doi. org/10.1016/0031-9163(65)90751-1http://linkinghub.elsevier.com/retrieve/pii/ 0031916365907511. 
[49] M. Nooijen and R. J. Bartlett, J. Chem. Phys. 102, 3629 (1995), ISSN 00219606, URL http: //link.aip.org/link/JCPSA6/v102/i9/p3629/s1\\&Agg=doi.

[50] M. Nooijen and R. J. Bartlett, J. Chem. Phys. 106, 6441 (1997), ISSN 00219606, URL http: //link.aip.org/link/JCPSA6/v106/i15/p6441/s1\\&Agg=doi.

[51] S. R. Gwaltney, R. J. Bartlett, and M. Nooijen, J. Chem. Phys. 111, 58 (1999), ISSN 00219606, URL http://link.aip.org/link/JCPSA6/v111/i1/p58/s1\\&Agg=doi.

[52] I. Shavitt and R. J. Bartlett, Many-Body Methods in Chemistry and Physics: MBPT and Coupled-Cluster Theory (Cambridge University Press, 2009).

[53] H. Sekino and R. J. Bartlett, Int. J. Quantum Chem 26, 255 (1984).

[54] K. Kowalski, J. R. Hammond, and W. A. de Jong, J. Chem. Phys. 127, 164105 (2007).

[55] M. E. Casida, in Recent advances in computational chemistry, edited by D. P. Chong (World Scientific, Singapore, 1995), vol. 1, p. 155.

[56] N. J. Higham, Functions of Matrices: Theory and Computation, SIAM e-books (Society for Industrial and Applied Mathematics (SIAM, 3600 Market Street, Floor 6, Philadelphia, PA 19104), 2008), ISBN 9780898717778, URL http://books.google.com/books?id= S6gpNn1JmbgC.

[57] R. G. Parr and W. Yang, Density-Functional Theory of Atoms And Molecules (Oxford University Press, New York, 1989).

[58] CFOUR, Coupled-Cluster techniques for Computational Chemistry, a quantum-chemical program package by J.F. Stanton, J. Gauss, M.E. Harding, P.G. Szalay with contributions from A.A. Auer, R.J. Bartlett, U. Benedikt, C. Berger, D.E. Bernholdt, Y.J. Bomble, L.

[59] An in-house program for QM/MM simulations (http://www.qm4d.info).

[60] T. H. Dunning, J. Chem. Phys. 90, 1007 (1989), ISSN 00219606, URL http://link.aip.org/ link/JCPSA6/v90/i2/p1007/s1\\&Agg=doi.

[61] D. E. Woon and T. H. Dunning, J. Chem. Phys. 98, 1358 (1993), ISSN 00219606, URL http://link.aip.org/link/JCPSA6/v98/i2/p1358/s1\\&Agg=doi.

[62] A. D. Becke, J. Chem. Phys. 98, 5648 (1993).

[63] C. T. Lee, W. T. Yang, and R. G. Parr, Phys. Rev. B 37, 785 (1988).

[64] J. P. Perdew, K. Burke, and M. Ernzerhof, Phys. Rev. Lett. 77, 3865 (1996).

[65] R. J. Bartlett, I. Grabowski, S. Hirata, and S. Ivanov, J. Chem. Phys. 122, 34104 (2005), ISSN 0021-9606, URL http://www.ncbi.nlm.nih.gov/pubmed/15740189. 
[66] R. J. Bartlett, V. F. Lotrich, and I. V. Schweigert, J. Chem. Phys. 123, 62205 (2005), ISSN 0021-9606, URL http://www.ncbi.nlm.nih.gov/pubmed/16122291.

[67] J. D. Watts, J. Gauss, and R. J. Bartlett, J. Chem. Phys. 98, 8718 (1993), URL http: //dx.doi.org/10.1063/1.464480.

[68] G. J. O. Beran, S. R. Gwaltney, and M. Head-Gordon, Phys. Chem. Chem. Phys. 5, 2488 (2003), ISSN 1463-9076, URL http://dx.doi.org/10.1039/B304542K.

[69] L. A. Curtiss, K. Raghavachari, P. C. Redfern, and J. A. Pople, J. Chem. Phys. 112, 7374 (2000).

[70] L. A. Curtiss, P. C. Redfern, and K. Raghavachari, J. Chem. Phys. 123, 124107 (2005), URL http://link. aip.org/link/?JCP/123/124107/1.

[71] P. Mori-Sánchez, A. Cohen, and W. Yang, Physical Review Letters 100, 146401 (2008), ISSN 0031-9007, URL http://link.aps.org/doi/10.1103/PhysRevLett.100.146401.

[72] J. D. Watts and R. J. Bartlett, J. Chem. Phys. 95, 6652 (1991), ISSN 00219606, URL http: //link. aip.org/link/JCPSA6/v95/i9/p6652/s1\\&Agg=doi.

[73] J. A. Pople, M. Head-Gordon, D. J. Fox, K. Raghavachari, and L. A. Curtiss, J. Chem. Phys. 90, 5622 (1989), ISSN 00219606, URL http://link.aip.org.proxy.lib.duke.edu/link/ ?JCPSA6/90/5622/1.

[74] L. A. Curtiss, C. Jones, G. W. Trucks, K. Raghavachari, and J. A. Pople, J. Chem. Phys. 93, 2537 (1990), ISSN 00219606, URL http://link.aip.org.proxy.lib.duke.edu/link/ ?JCPSA6/93/2537/1.

[75] L. A. Curtiss, K. Raghavachari, P. C. Redfern, and J. A. Pople, J. Chem. Phys. 106, 1063 (1997), ISSN 00219606, URL http://link.aip.org.proxy.lib.duke.edu/link/?JCPSA6/ $106 / 1063 / 1$.

[76] C. Adamo, M. Ernzerhof, and G. E. Scuseria, J. Chem. Phys. 112, 2643 (2000), ISSN 00219606, URL http://link.aip.org/link/?JCPSA6/112/2643/1http://link.aip. org/link/JCPSA6/v112/i6/p2643/s1 \&Agg=doi.

[77] Note that the expression in Ref. [7] corresponding to the second equation in Eq. (16) is wrong. The correct expression is present in Ref. [? ]. 


\section{Appendix A: Mathematical analysis of the pp-RPA equation}

The appendix discusses many mathematical properties of the pp-RPA equation. These properties are conceptually very similar to those of ph-RPA equation as shown in Ref. [10].

\section{The zero signature of an eigenvector with an imaginary eigenvalue}

For an eigenvalue $\omega_{n}$ and eigenvector $\mathbf{z}_{n}$, we have

$$
\mathbf{M} \mathbf{z}_{n}=\omega_{n} \mathbf{W} \mathbf{z}_{n}
$$

The Hermitian conjugate of Eq. (A1) becomes

$$
\mathbf{z}_{n}^{\dagger} \mathbf{M}=\omega_{n}^{*} \mathbf{z}_{n}^{\dagger} \mathbf{W}
$$

Multiplying $\mathbf{z}_{n}^{\dagger}$ to the left of Eq. (A1) and $\mathbf{z}_{n}$ to the right of Eq. (A2), we have

$$
\mathbf{z}_{n}^{\dagger} \mathbf{M} \mathbf{z}_{n}=\omega_{n} \mathbf{z}_{n}^{\dagger} \mathbf{W} \mathbf{z}_{n}=\omega_{n}^{*} \mathbf{z}_{n}^{\dagger} \mathbf{W} \mathbf{z}_{n}
$$

Therefore

$$
\left(\omega_{n}-\omega_{n}^{*}\right)\left(\mathbf{z}_{n}^{\dagger} \mathbf{W} \mathbf{z}_{n}\right)=0
$$

For an imaginary eigenvalue $\omega_{n} \neq \omega_{n}^{*}$, the signature $\mathbf{z}_{n}^{\dagger} \mathbf{W} \mathbf{z}_{n}=0$.

\section{The orthonormalization of eigenvectors with all real eigenvalues}

Using the same approach in Subsection A 1 in Appendix but with two different eigenvalues and eigenvectors, we have

$$
\mathbf{z}_{n}^{\dagger} \mathbf{M} \mathbf{z}_{m}=\omega_{m} \mathbf{z}_{n}^{\dagger} \mathbf{W} \mathbf{z}_{m}=\omega_{n}^{*} \mathbf{z}_{n}^{\dagger} \mathbf{W} \mathbf{z}_{m}
$$

and

$$
\left(\omega_{m}-\omega_{n}^{*}\right)\left(\mathbf{z}_{n}^{\dagger} \mathbf{W} \mathbf{z}_{m}\right)=0
$$

Therefore, when two real eigenvalues are different $\left(\omega_{m} \neq \omega_{n}^{*}\right)$, the two eigenvectors are orthogonal under the metric $\mathbf{W}\left(\mathbf{z}_{n}^{\dagger} \mathbf{W} \mathbf{z}_{m}=0\right)$. Since linear combination of eigenvectors of a degenerate eigenvalue stays in the same eigenspace, we can choose the eigenvectors of a degenerate eigenvalue to orthogonal to each other within the eigenspace. When all 
eigenvalues are real, eigenvectors can, therefore, be chosen to be orthogonalized under the metric $\mathbf{W}$. For a diagonalizable pp-RPA equation with all real eigenvalues, $\mathbf{z}_{n}^{\dagger} \mathbf{W} \mathbf{z}_{n}$ should not be zero, otherwise we have $\mathbf{z}_{n}^{\dagger} \mathbf{W Z}=0$, which indicates the eigenvector matrix is rankdeficit, which contradicts with the diagonalizability assumption. Therefore, the signatures of eigenvectors are all nonzero for a diagonalizable pp-RPA equation with all real eigenvalues. The resulting orthonormalization can be written as

$$
\mathbf{Z}^{\dagger} \mathbf{W Z}=\Lambda,
$$

where $\Lambda$ is a diagonal matrix with only \pm 1 diagonal elements. According to Sylvester's law of inertia[? ], $\mathbf{W}$ and $\Lambda$ share the same number of +1 's and -1 's. In another word, there are $N_{p p} N+2$ excitations and $N_{h h} N-2$ excitations, according to the definition of $N \pm 2$ excitations in Sec. III. We can further arrange the eigenvectors such that eigenvectors with positive signatures stay in the left of $\mathbf{Z}$, then finally we reach the normalization condition

$$
\mathbf{Z}^{\dagger} \mathbf{W Z}=\mathbf{W}
$$

\section{The equivalence between stability and positive definiteness of $\mathbf{M}$}

First we show that the stability condition of Eq. (13) leads to the positive definiteness of $\mathrm{M}$.

From the stability of the pp-RPA equation (Eq. (13)) and the normalization (Eq. (12)), we have

$$
\begin{aligned}
\mathbf{c}^{\dagger} \mathbf{M c} & =\sum_{m n}\left(\mathbf{z}_{m} c_{m}\right)^{\dagger} \mathbf{M}\left(\mathbf{z}_{n} c_{n}\right) \\
& =\sum_{m n} c_{m}^{*} \mathbf{z}_{m}^{\dagger} \omega_{n} \mathbf{W} \mathbf{z}_{n} c_{n} \\
& =\sum_{n} c_{m}^{*} \delta_{m n} W_{m n} \omega_{n} c_{n} \\
& =\sum_{m n} c_{m}^{*}\left|\omega_{m}\right| \delta_{m n} c_{n} \\
& =\sum_{m}\left|c_{m}\right|^{2}\left|\omega_{m}\right|>0
\end{aligned}
$$

with an arbitrary nonzero column vector $\mathbf{c}$. Thus, $\mathbf{M}$ is positive definite for a pp-RPA equation. 
Next, we show that the reverse is also true.

Given that $\mathbf{M}$ is positive definite, the pp-RPA equation in the compact form reads

$$
\mathbf{M} \mathbf{z}_{n}=\omega_{n} \mathbf{W} \mathbf{z}_{n}
$$

Since $\mathbf{M}$ is positive definite, Eq. (8) could be rewritten as

$$
\mathbf{L}^{\dagger} \mathbf{z}_{n}=\omega_{n} \mathbf{L}^{-1} \mathbf{W}\left(\mathbf{L}^{-1}\right)^{\dagger} \mathbf{L}^{\dagger} \mathbf{z}_{n}
$$

where $\mathbf{M}=\mathbf{L} \mathbf{L}^{\dagger}$ is the Cholesky decomposition. With $\tilde{\mathbf{z}}_{n}=\mathbf{L}^{\dagger} \mathbf{z}_{n}$ and $\tilde{\mathbf{W}}=\mathbf{L}^{-1} \mathbf{W}\left(\mathbf{L}^{-1}\right)^{\dagger}$, then the eigenvalue problem

$$
\tilde{\mathbf{W}} \tilde{\mathbf{z}}_{n}=\tilde{\omega}_{n} \tilde{\mathbf{z}}_{n}
$$

is diagonalizable with all real eigenvalues, since $\tilde{\mathbf{W}}^{\dagger}=\tilde{\mathbf{W}}$ by definition. Additionally, all eigenvalues of $\tilde{\mathbf{W}}, \tilde{\omega}_{n}{ }^{\prime}$ s, will be nonzero, since zero eigenvalue indicates $\operatorname{det}(\tilde{\mathbf{W}})=0$ which contradicts the definition of $\tilde{\mathbf{W}}$. With orthonormalization of the eigenvectors $\tilde{\mathbf{z}}_{n}^{\dagger} \tilde{\mathbf{z}}_{m}=$ $\delta_{n m}\left|\tilde{\omega}_{n}\right|^{-1}$, Eq. (8) can be diagonalized with real eigenvalues

$$
\omega_{n}=\tilde{\omega}_{n}^{-1}
$$

and eigenvector orthonormalization with the eigenvalue sign constraints (the eigenvectors are arranged in the same way as in Subsection A 2 in Appendix),

$$
\mathbf{z}_{n}^{\dagger} \mathbf{W} \mathbf{z}_{m}=\delta_{m n} \operatorname{sign}\left(\omega_{m}\right)=W_{n m}
$$

Eq. (A10) guarantees that the $\min _{n} \omega_{n}^{N+2}>0>\max _{m} \omega_{m}^{N-2}$. Therefore, by definition, this pp-RPA equation is stable since all the eigenvalues are real and the $N+2$ and $N-2$ excitation spectra are nicely separated.

In summary, the stability condition of an pp-RPA equation is equivalent to the positive definiteness of $\mathbf{M}$.

\section{The invertibility of $\mathrm{X}$ for a stable pp-RPA equation}

We now prove the invertibility of $\mathbf{X}$ in Sec. IV. According to Subsection A 2 in Appendix, the eigenvalues of a stable pp-RPA equation are orthonormalized according to

$$
\mathbf{Z}^{\dagger} \mathbf{W Z}=\mathbf{W}
$$


For only $N+2$ excitation vectors,

$$
\mathbf{Z}_{N+2}^{\dagger} \mathbf{W} \mathbf{Z}_{N+2}=\mathbf{I}
$$

where

$$
\mathbf{Z}_{N+2}=\left[\begin{array}{l}
\mathbf{X} \\
\mathbf{Y}
\end{array}\right]
$$

with $\mathbf{X}$ and $\mathbf{Y}$ the particle-particle and hole-hole block of the $N+2$ excitation eigenvector matrices. Expanding Eq. (A12), we have

$$
\mathbf{X}^{\dagger} \mathbf{X}-\mathbf{Y}^{\dagger} \mathbf{Y}=\mathbf{I}
$$

Therefore, $\mathbf{X}^{\dagger} \mathbf{X}=\mathbf{I}+\mathbf{Y}^{\dagger} \mathbf{Y}$ is positive definite, and $\mathbf{X}$ is invertible, otherwise $\mathbf{X}^{\dagger} \mathbf{X}$ will not be positive definite. 